\title{
Wedge locality and asymptotic commutativity
}

\author{
M. A. Soloviev* \\ I. E. Tamm Department of Theoretical Physics, P. N. Lebedev Physical Institute, \\ Russian Academy of Sciences, Leninsky Prospect 53, Moscow 119991, Russia
}

\begin{abstract}
In this paper, we study twist deformed quantum field theories obtained by combining the Wightman axiomatic approach with the idea of spacetime noncommutativity. We prove that the deformed fields with deformation parameters of opposite sign satisfy the condition of mutual asymptotic commutativity, which was used earlier in nonlocal quantum field theory as a substitute for relative locality. We also present an improved proof of the wedge localization property discovered for the deformed fields by Grosse and Lechner, and we show that the deformation leaves the asymptotic behavior of the vacuum expectation values in spacelike directions substantially unchanged.
\end{abstract}

PACS numbers: 11.10.Nx, 03.65.Db, 03.70.+k, 11.10.Cd

\section{INTRODUCTION}

Models of quantum field theory on noncommutative spacetime continue to attract attention because of their relevance for understanding quantum gravity [1] and because they can be obtained as a particular low-energy limit of string theory [2]. Noncommutativity is usually introduced by replacing the spacetime coordinates $x^{\mu}$ with Hermitian operators $\hat{x}^{\mu}$ satisfying commutation relations of the form

$$
\left[x^{\mu}, x^{\nu}\right]=i \theta^{\mu \nu}
$$

where $\theta^{\mu \nu}$ is a real antisymmetric matrix, constant in the simplest case. The relations (1) are translation invariant, but not Lorentz covariant. The twist deformation was devised [3, 4] as a way to restore the spacetime symmetries broken by noncommutativity. In its widest form [5], the twisting principle implies that all symmetries and products of the theory should be consistently deformed by properly applying a twist operator. In particular, the tensor product $f \otimes g$ of two functions on spacetime is deformed in the following way: $f \otimes g \rightarrow f \otimes_{\theta} g$,

*Electronic address: soloviev@lpi.ru 
where

$$
\left(f \otimes_{\theta} g\right)(x, y) \stackrel{\text { def }}{=} \exp \left(\frac{i}{2} \theta^{\mu \nu} \frac{\partial}{\partial x^{\mu}} \frac{\partial}{\partial y^{\mu}}\right) f(x) g(y),
$$

and the twist operator here is

$$
\mathcal{T}=e^{\frac{i}{2} \theta^{\mu \nu} \partial_{\mu} \otimes \partial_{\nu}}
$$

(Hereafter, we use the usual summation convention for the repeated indices.) From the standpoint of deformation quantization, noncommutativity amounts to deforming the ordinary pointwise product $f(x) g(x)$ to the Weyl-Moyal star product $f \star_{\theta} g$ which is obtained from $f \otimes_{\theta} g$ by restricting to the diagonal,

$$
\left(f \star_{\theta} g\right)(x)=\left(f \otimes_{\theta} g\right)(x, x) .
$$

For the coordinate functions, we have

$$
\left[x^{\mu}, x^{\nu}\right]_{\star} \equiv x^{\mu} \star x^{\nu}-x^{\nu} \star x^{\mu}=i \theta^{\mu \nu},
$$

which is related to (1) by the Weyl-Wigner correspondence. The strategy of twisting also leads to deformed commutation relations for the creation and annihilation operators of free fields, see [5-10]. However, as shown in [8, 10, 11], some combinations of twistings can cancel noncommutativity. Then the S-matrix of twisted quantum field theory turns out to be equivalent to that of its commutative counterpart, and this issue does not seem completely resolved (compare, e.g., [12] and [13]).

A new interesting line of research concerns the use of noncommutative deformations of free field theories as a means of constructing integrable models with a factorizable S-matrix. Grosse and Lechner [14, 15] studied a deformation of this type, generated by twisting the tensor algebra of test functions in the Wightman framework [16], and they discovered that the deformed fields can be localized in wedge-shaped regions of Minkowski space. Grosse and Lechner also showed that the deformation introduces a nontrivial interaction and that this weak form of locality is sufficient for computing two-particle S-matrix elements. A more general deformation techniques have been developed in an operator-algebraic setting [17, 18] and then extended to quantum field theory on a curved spacetime [19]. This deformation method was also applied to a fermionic model [20] and was used to construct wedge-local fields with anyonic statistics [21].

In this paper, we consider the twisted quantum field theory from a complementary point of view stated in [22, 23], with emphasis on the nonlocal aspects of the deformation. The 
deformation procedure described below in terms of the Wightman functions applies to interacting as well as free fields. Our main observation is that the fields $\phi^{\theta}$ and $\phi^{-\theta}$ with deformation parameters of opposite sign satisfy the condition of mutual asymptotic commutativity, which was used earlier in nonlocal quantum field theory (see, e.g., [24] and references therein) as an analog of relative locality. This result supplements the wedge localization property found in [14, 15]. We also show that the deformation does not spoil the asymptotic behavior of the vacuum expectation values in spacelike directions, which plays the major role in constructing the asymptotic scattering states in the deformed theory.

The paper is organized as follows. In Sec. III, we list basic properties of the twisted tensor product $\otimes_{\theta}$ and define the corresponding deformation of Wightman functions. In Sec. III, we present an improved proof of the wedge locality property of the deformed fields. In Sec. IV, we obtain a characterization of the asymptotic behavior of the (anti)commutator $\left[\phi^{\theta}(x), \phi^{-\theta}(y)\right]_{(+)}$of two fields with deformation parameters of opposite sign. We show that this commutator falls off rapidly at large spacelike separation of $x$ and $y$, and we estimate the fall-off rate. Particular attention is given to the adequate choice of the test functions that are required for this purpose. At this point, we use a criterion [25] under which a test function space has the structure of an algebra with respect to the Weyl-Moyal star product. Our analysis shows, in particular, that the commutator under study satisfies the asymptotic commutativity condition proposed for nonlocal fields in [26]. In Sec. $\mathrm{Z}$, we prove that the deformation has little or no effect on the asymptotic behavior of the vacuum expectation values in spacelike directions. Section VI contains concluding remarks.

\section{TWIST DEFORMATION OF WIGHTMAN FUNCTIONS}

In order that the twisted tensor product (21) and Weyl-Moyal star product (4) be well defined, the functions involved must satisfy certain conditions. In quantum field theory formalism, it is a standard practice to use the Schwartz space $S$ of smooth functions decreasing faster than any inverse power of their arguments, and this space is, as well known, an algebra under the star multiplication. But it should be kept in mind that the expansions of both these products in powers of the noncommutative parameter $\theta$ are in general divergent for functions in $S$. A preferable definition of these products is by using the Fourier transformation, which converts the twist operator (3) to the multiplication by the function

$$
\eta(p, q)=e^{-\frac{i}{2} p \theta q}, \quad \text { where } \quad p \theta q \stackrel{\text { def }}{=} p_{\mu} \theta^{\mu \nu} q_{\nu} .
$$


The function $\eta$ is a multiplier of the Schwartz space and $\widehat{f \otimes_{\theta} g}$ may be written as

$$
\left(\widehat{f \otimes_{\theta} g}\right)(p, q)=e^{-\frac{i}{2} p \theta q} \hat{f}(p) \hat{g}(q) .
$$

This definition extends to the case of several variables in the following way

$$
\begin{aligned}
\left(f_{(m)} \widehat{\otimes_{\theta}} g_{(n)}\right)\left(p_{1}, \ldots p_{m} ; q_{1}, \ldots, q_{n}\right)= & \\
& =\prod_{j=1}^{m} \prod_{k=1}^{n} e^{-\frac{i}{2} p_{j} \theta q_{k}} \hat{f}_{(m)}\left(p_{1}, \ldots p_{m}\right) \hat{g}_{(n)}\left(q_{1}, \ldots, q_{n}\right),
\end{aligned}
$$

where $f_{m}$ and $g_{n}$ are assumed to be elements of $S\left(\mathbb{R}^{4 m}\right)$ and $S\left(\mathbb{R}^{4 n}\right)$ respectively. It is easy to see that the bilinear map $(f, g) \rightarrow f \otimes_{\theta} g$ is continuous in the topology of the Schwartz space and satisfies the associativity condition $f \otimes_{\theta}\left(g \otimes_{\theta} h\right)=\left(f \otimes_{\theta} g\right) \otimes_{\theta} h$, which really determines the form of the multiplier in (8) .

We now turn to the noncommutative deformation [14, 15, 22] of quantum field theories that can be associated with the twisted tensor product. Let $\left\{\phi_{\iota}\right\}_{\iota \in I}$ be a finite system of quantum fields transforming according to irreducible finite-dimensional representations of the proper Lorentz group $L_{+}^{\uparrow}$ or its covering group $S L(2, \mathbb{C})$. Their components are labelled by an additional index $l$, but for brevity we let $\iota$ denote the pair $(\iota, l)$. We suppose that all the assumptions of the Wightman formulation [16] of local quantum field theory are satisfied and $\phi_{\iota}$ are defined as operator-valued distributions with a common dense invariant domain in a Hilbert space $\mathcal{H}$. As usual, we denote by $\Omega$ the vacuum state, by $w_{\iota_{1} \ldots \iota_{n}}$ the vacuum expectation value of a product of $n$ fields, and identify it with a tempered distribution on $\mathbb{R}^{4 n}$

$$
\left\langle\Omega, \phi_{\iota_{1}}\left(f_{1}\right) \cdots \phi_{\iota_{n}}\left(f_{n}\right) \Omega\right\rangle=w_{\iota_{1} \ldots \iota_{n}}\left(f_{1} \otimes \cdots \otimes f_{n}\right), \quad w_{\iota_{1} \ldots \iota_{n}} \in S^{\prime}\left(\mathbb{R}^{4 n}\right) .
$$

The deformed Wightman functions $w_{(\ldots)}^{\theta}$ are defined by

$$
w_{\iota_{1} \ldots \iota_{n}}^{\theta}\left(f_{1} \otimes \cdots \otimes f_{n}\right) \stackrel{\text { def }}{=} w_{\iota_{1} \ldots \iota_{n}}\left(f_{1} \otimes_{\theta} \cdots \otimes_{\theta} f_{n}\right), \quad f_{j} \in S\left(\mathbb{R}^{4}\right),
$$

or equivalently, by

$$
\hat{w}_{\iota_{1} \ldots \iota_{n}}^{\theta}=\prod_{1 \leq j<k \leq n} e^{-\frac{i}{2} p_{j} \theta p_{k}} \hat{w}_{\iota_{1} \ldots \iota_{n}} .
$$

The set of deformed distributions $w_{(\ldots)}^{\theta}$ satisfies the positive-definiteness condition (see [22]). Furthermore, as shown below in Sec. V], the deformation (10) does not spoil the cluster decomposition property, and so if $a$ is a spacelike vector, then for any $f \in S\left(\mathbb{R}^{4 m}\right)$ and $g \in S\left(\mathbb{R}^{4(n-m)}\right)$, the following relation holds:

$$
w_{\iota_{1} \ldots \iota_{n}}^{\theta}\left(f \otimes g_{(\lambda a)}\right) \longrightarrow w_{\iota_{1} \ldots \iota_{m}}^{\theta}(f) w_{\iota_{m+1} \ldots \iota_{n}}^{\theta}(g) \quad \text { as } \quad \lambda \rightarrow \infty
$$


where by $g_{(\lambda a)}$ we mean the shifted function, i.e., $g_{(\lambda a)}\left(x_{m+1}, \ldots, x_{n}\right)=g\left(x_{m+1}-\lambda a, \ldots, x_{n}-\right.$ $\lambda a)$. Therefore, by the Wightman reconstruction theorem [16], this set of distributions determines a field theory uniquely, up to unitary equivalence. It is easy to construct explicitly quantum fields $\phi_{\iota}^{\theta}$ having such expectation values. As shown in [16], the Schwartz kernel theorem gives a precise meaning to vectors of the form

$$
\Phi_{\iota_{1} \ldots \iota_{n}}(g)=\int d x_{1} \ldots d x_{n} g\left(x_{1}, \ldots, x_{n}\right) \phi_{\iota_{1}}\left(x_{1}\right) \cdots \phi_{\iota_{n}}\left(x_{n}\right) \Omega, \quad \text { where } g \in S\left(\mathbb{R}^{4 n}\right),
$$

and the linear subspace $D$ spanned by all these vectors and $\Omega$ can be taken as a common domain of the initial fields $\phi_{\iota}$. For each $f \in S\left(\mathbb{R}^{4}\right)$, we define $\phi_{\iota}^{\theta}(f)$ by

$$
\phi_{\iota}^{\theta}(f) \Omega=\phi_{\iota}(f) \Omega, \quad \phi_{\iota}^{\theta}(f) \Phi_{\iota_{1} \ldots \iota_{n}}(g)=\Phi_{\iota \iota_{1} \ldots \iota_{n}}\left(f \otimes_{\theta} g\right), \quad n \geq 1,
$$

extended by linearity.

It is easy to verify that the fields $\phi_{\iota}^{\theta}(f)$ are well defined as operator-valued tempered distributions with the same common domain $D \subset \mathcal{H}$, and it is clear that

$$
\left\langle\Omega, \phi_{\iota_{1}}^{\theta}\left(f_{1}\right) \cdots \phi_{\iota_{1}}^{\theta}\left(f_{n}\right) \Omega\right\rangle=w_{\iota_{1} \ldots \iota_{n}}^{\theta}\left(f_{1} \otimes \cdots \otimes f_{n}\right) .
$$

We also note that the linear span of all vectors of the form analogous to (13) but with $\phi_{\iota_{j}}^{\theta}$ in place of $\phi_{\iota_{j}}$ coincides with $D$, because the multiplier $\prod_{j<k} e^{-\frac{i}{2} p_{j} \theta p_{k}}$ maps $S\left(\mathbb{R}^{4 n}\right)$ isomorphically onto itself.

The basic properties of the deformed Wightman functions and fields are described in 14, 15] and in 22, 23] for the case of a single neutral scalar field. The deformation does not change the support properties of the vacuum expectation values in the momentum space, and therefore the distributions $w_{(\ldots)}^{\theta}$ satisfy the spectrum condition. The translation invariance is also preserved. The vacuum $\Omega$ is a cyclic vector for the deformed fields $\phi_{l}^{\theta}$. Moreover, as pointed out in [15], they have the Reeh-Schlieder property, i.e., for each nonempty open set $O \subset \mathbb{R}^{4}$, the linear span of vectors of the form $\prod_{j=1}^{n} \phi_{\iota_{j}}^{\theta}\left(f_{j}\right) \Omega$ with supp $f_{j} \subset O$ is dense in $\mathcal{H}$. If a field $\phi_{\iota}$ is Hermitian, then so is $\phi_{\iota}^{\theta}$. The derivation of these properties uses in an essential way the identity

$$
w_{(\ldots)}\left(f \otimes_{\theta} g\right)=w_{(\ldots)}(f \otimes g),
$$

which holds for every $n$-point Wightman function and for any test functions $f \in S\left(\mathbb{R}^{4 m}\right)$ and $g \in S\left(\mathbb{R}^{4(n-m)}\right)$, where $1<m<n$. The identity (15) follows directly from (8) and the translation invariance of the distributions $w_{(\ldots)}$, because the matrix $\theta^{\mu \nu}$ is antisymmetric. In 
the case of a free neutral scalar field $\phi$, its creation and annihilation operators are deformed as follows

$$
a_{\theta}(p)=e^{\frac{i}{2} p \theta P} a(p), \quad a_{\theta}^{*}(p)=e^{-\frac{i}{2} p \theta P} a^{*}(p),
$$

where $P$ is the energy-momentum operator. The operators $a_{\theta}(p)$ and $a_{\theta}^{*}(p)$ satisfy the deformed canonical commutation relations discussed in [5 10]. As already noted, the deformation (10) preserves the translation invariance, but it violates the Lorentz covariance and the fields $\phi_{\iota}^{\theta}$ transform covariantly only under those Lorentz transformations that leave the matrix $\theta^{\mu \nu}$ unaltered. This deformation also leads to a strong violation of locality, and the fields $\phi_{\iota}^{\theta}$ do not satisfy the microcausality condition. This is easily seen by considering matrix elements of the deformed field commutator in the simplest case of a free scalar field. Theorem 3 of [22] shows that if $\theta^{\mu \nu} \neq 0$, then the matrix elements of the form $\left\langle\Omega,\left[\phi^{\theta}(x), \phi^{\theta}(y)\right] \Phi\right\rangle$, where $\Phi$ is a normalized two-particle state, are nonzero everywhere, i.e., their supports coincide with $\mathbb{R}^{4} \times \mathbb{R}^{4}$. It should be noted that this is also true for the case of so-called space-space noncommutativity, where $\theta^{0 \nu}=0$ for all $\nu$. Therefore the deformed fields $\phi_{\iota}^{\theta}$ do not satisfy even the relaxed local commutativity condition [13, 27, 28] adapted to this case and obtained by replacing the light cone with the light wedge. Nevertheless the fields $\phi_{\iota}^{\theta}$ are not completely delocalized, and because the issues of locality and causality are crucial for the physical interpretation, the remainder of the paper is devoted to a precise description of the extent to which the noncommutative deformation violates locality and local commutativity.

\section{WEDGE LOCALITY}

From the definition (8) of the deformed tensor product, it directly follows that, for any $f_{1}, f_{2} \in S\left(\mathbb{R}^{4}\right)$ and $g \in S\left(\mathbb{R}^{4 n}\right)$, the following identity holds:

$$
\left(f_{1} \otimes_{\theta}\left(f_{2} \otimes_{-\theta} g\right)\right)\left(x_{1}, x_{2}, y\right)=\left(f_{2} \otimes_{-\theta}\left(f_{1} \otimes_{\theta} g\right)\right)\left(x_{2}, x_{1}, y\right)
$$

where $y=\left(y_{1}, \ldots y_{n}\right)$. Indeed, let $p_{1}, p_{2}$, and $q_{j}$ be the variables conjugate respectively to $x_{1}, x_{2}$, and $y_{j}$, and let $Q=\sum_{j=1}^{n} q_{j}$. The Fourier transform of the left-hand side of (16) is $\left(\hat{f}_{1} \otimes \hat{f}_{2} \otimes \hat{g}\right)\left(p_{1}, p_{2}, q\right)$ multiplied by $\exp \left\{-\frac{i}{2}\left(p_{1} \cdot \theta p_{2}+p_{1} \cdot \theta Q-p_{2} \cdot \theta Q\right)\right\}$, and that of the right-hand side is obtained by multiplication with $\exp \left\{-\frac{i}{2}\left(-p_{2} \cdot \theta p_{1}-p_{2} \cdot \theta Q+p_{1} \cdot \theta Q\right)\right\}$. Clearly, these two multipliers coincide because the matrix $\theta^{\mu \nu}$ is antisymmetric.

We will also use the following fact. If $f \in S\left(\mathbb{R}^{4}\right), g \in S\left(\mathbb{R}^{4 n}\right)$, and $f$ has compact support, 
then we have the inclusions ${ }^{1}$

$$
\operatorname{supp}\left(f \otimes_{\theta} g\right) \subset\left(\operatorname{supp} f-\frac{1}{2} \theta U_{\hat{g}}\right) \times \mathbb{R}^{4 n}, \quad \operatorname{supp}\left(g \otimes_{\theta} f\right) \subset \mathbb{R}^{4 n} \times\left(\operatorname{supp} f+\frac{1}{2} \theta U_{\hat{g}}\right),
$$

where $U_{\hat{g}}$ is the closure of the set $\left\{Q \in \mathbb{R}^{4}: Q=\sum_{j=1}^{n} q_{j},\left(q_{1}, \ldots, q_{n}\right) \in \operatorname{supp} \hat{g}\right\}$. Indeed, (8) implies that

$$
\begin{aligned}
\left(f \otimes_{\theta} g\right)(x, y)=(2 \pi)^{-4(n+1)} \int \hat{f}(p) \hat{g}(q) e^{-i p \cdot x-i \sum_{j=1}^{n} q_{j} \cdot y_{j}-\frac{i}{2} p \cdot \theta Q} d p d q_{1} \ldots d q_{n} \\
=(2 \pi)^{-4 n} \int f\left(x+\frac{1}{2} \theta Q\right) \hat{g}(q) e^{-i \sum_{j=1}^{n} q_{j} \cdot y_{j}} d q_{1} \ldots d q_{n} .
\end{aligned}
$$

This integral is nonzero only if $x+\frac{1}{2} \theta \sum_{j=1}^{n} q_{j}$ belongs to supp $f$ for some $\left(q_{1}, \ldots, q_{n}\right) \in \operatorname{supp} \hat{g}$; hence $x \in \operatorname{supp} f-\frac{1}{2} \theta U_{\hat{g}}$. The latter set is closed because the support of $f$ is assumed to be compact. The second inclusion in (17) is proved analogously. Now let $f_{1}, f_{2} \in S\left(\mathbb{R}^{4}\right)$, $g \in S\left(\mathbb{R}^{4 n}\right), h \in S\left(\mathbb{R}^{4 m}\right)$, and let $f_{1}$ and $f_{2}$ be of compact support. Then it follows from (17) that

$$
\operatorname{supp}\left(h \otimes_{\theta} f_{1}\right) \otimes\left(f_{2} \otimes_{-\theta} g\right) \subset \mathbb{R}^{4 m} \times\left(\operatorname{supp} f_{1}+\frac{1}{2} \theta U_{\hat{h}}\right) \times\left(\operatorname{supp} f_{2}+\frac{1}{2} \theta U_{\hat{g}}\right) \times \mathbb{R}^{4 n} .
$$

Following [14, 15], we introduce the reference matrix

$$
\theta_{1}=\left(\begin{array}{cccc}
0 & \vartheta_{e} & 0 & 0 \\
-\vartheta_{e} & 0 & 0 & 0 \\
0 & 0 & 0 & \vartheta_{m} \\
0 & 0 & -\vartheta_{m} & 0
\end{array}\right),
$$

where $\vartheta_{e} \geq 0$ and $\vartheta_{m} \neq 0$, and we let $\mathrm{W}_{1}$ denote the right-hand wedge in Minkowski space, defined by

$$
\mathrm{W}_{1}=\left\{x \in \mathbb{R}^{4}: x^{1}>\left|x^{0}\right|\right\} .
$$

As shown in [14], the stabilizer subgroup of the matrix $\theta_{1}$ with respect to the action $\theta \rightarrow$ $\Lambda \theta \Lambda^{T}$ of the proper orthochronous Lorentz group $L_{+}^{\uparrow}$ coincides with that of the wedge $\mathrm{W}_{1}$ with respect to the action $\mathrm{W} \rightarrow \Lambda \mathrm{W}$, and there is therefore a one-to-one correspondence between the orbits of $\theta_{1}$ and $\mathrm{W}_{1}$. It is easy to see that if a matrix $\theta$ belongs to the orbit of $\theta_{1}$ and $\mathrm{W}_{\theta}$ is its corresponding wedge, then $-\theta$ also belongs to this orbit and $\mathrm{W}_{-\theta}=-\mathrm{W}_{\theta}$.

\footnotetext{
$\overline{1}$ The definition of the Fourier transform used here is the same as in [29] and differs from that in [15] by a sign in exponent, and in consequence the signs in (17) differ from those in Eq. (3.12) of [15].
} 
Theorem 1 . (cf. Theorem 4.5 in [15]) Suppose that $\phi_{\iota}$ and $\phi_{\iota^{\prime}}$ belong to a set of Wightman fields with the common domain of definition D spanned by vectors of the form (13). Let $\theta=\Lambda \theta_{1} \Lambda^{T}$ and $\mathrm{W}_{\theta}=\Lambda \mathrm{W}_{1}$, where $\Lambda \in L_{+}^{\uparrow}$ and where $\theta_{1}$ and $\mathrm{W}_{1}$ are defined, respectively, by (201) and (21). If $\phi_{\iota}$ and $\phi_{\iota^{\prime}}$ (anti) commute at spacelike separation, ${ }^{2}$ then the deformed fields $\phi_{\iota}^{\theta}$ and $\phi_{\iota^{\prime}}^{\theta}$ satisfy the (anti)commutation relation

$$
\left[\phi_{\iota}^{\theta}\left(f_{1}\right), \phi_{\iota^{\prime}}^{-\theta}\left(f_{2}\right)\right]_{(+)} \Phi=0
$$

for all $\Phi \in D$ and for any $f_{1}, f_{2} \in S\left(\mathbb{R}^{4}\right)$ such that supp $f_{1} \subset \mathrm{W}_{\theta}$ and $\operatorname{supp} f_{2} \subset-\mathrm{W}_{\theta}$.

Proof. We consider the case when $\phi_{\iota}$ and $\phi_{\iota^{\prime}}$ commute at spacelike separation. Let $\Phi_{\iota_{1} \ldots \iota_{n}}(g)$ be a vector of the form (13), where $g \in S\left(\mathbb{R}^{4 n}\right)$, and let $\Phi_{(m)}(h)$ be a vector of an analogous form defined by a system of fields $\phi_{\iota_{1}^{\prime}}, \ldots, \phi_{\boldsymbol{\iota}_{m}^{\prime}}$ and a function $h \in S\left(\mathbb{R}^{4 m}\right)$. By the cyclicity of the vacuum, it suffices to show that the assumptions on the supports of $f_{1}$ and $f_{2}$ imply that

$$
\left\langle\Phi_{(m)}(h),\left[\phi_{\iota}^{\theta}\left(f_{1}\right), \phi_{\iota^{\prime}}^{-\theta}\left(f_{2}\right)\right] \Phi_{\iota_{1} \ldots \iota_{n}}(g)\right\rangle=0 .
$$

Furthermore, because this matrix element is continuous in $f_{1}$ and $f_{2}$ and smooth functions of compact support are dense in $S$, we can assume without loss of generality that $\operatorname{supp} f_{1}$ and supp $f_{2}$ are compact. Using the identity (16), this matrix element can be written as

$$
\left\langle\Phi_{(m)}(h),\left[\phi_{\iota}^{\theta}\left(f_{1}\right), \phi_{\iota^{\prime}}^{-\theta}\left(f_{2}\right)\right] \Phi_{\iota_{1} \ldots \iota_{n}}(g)\right\rangle=\left(w-w_{\pi}, h^{*} \otimes\left(f_{1} \otimes_{\theta}\left(f_{2} \otimes_{-\theta} g\right)\right)\right),
$$

where $w_{\pi}$ is obtained from $w$ by the transposition of the operators $\phi_{\boldsymbol{\iota}}\left(x_{1}\right)$ and $\phi_{\iota^{\prime}}\left(x_{2}\right)$, and where $h^{*}\left(z_{1}, \ldots, z_{m}\right)=\overline{h\left(z_{m}, \ldots, z_{1}\right)}$. Using (15) and the associativity of $\otimes_{\theta}$, we obtain

$$
\begin{aligned}
&\left(w-w_{\pi}, h^{*} \otimes\left(f_{1} \otimes_{\theta}\left(f_{2} \otimes_{-\theta} g\right)\right)\right)=\left(w-w_{\pi}, h^{*} \otimes_{\theta}\left(f_{1} \otimes_{\theta}\left(f_{2} \otimes_{-\theta} g\right)\right)\right)= \\
&\left(w-w_{\pi},\left(h^{*} \otimes_{\theta} f_{1}\right) \otimes\left(f_{2} \otimes_{-\theta} g\right)\right) .
\end{aligned}
$$

Let $k=\left(k_{1}, \ldots, k_{m}\right), p=\left(p_{1}, p_{2}\right)$, and $q=\left(q_{1}, \ldots, q_{n}\right)$ be the momentum variables conjugate to the coordinates on $\mathbb{R}^{4 m} \times \mathbb{R}^{4 \cdot 2} \times \mathbb{R}^{4 n}$. It follows from the spectrum condition that

$$
\operatorname{supp}\left(\hat{w}-\hat{w}_{\pi}\right) \subset\left\{(k, p, q) \in \mathbb{R}^{4(m+2+n)}: \sum_{j=1}^{m} k_{j} \in \bar{V}^{+}, \quad \sum_{j=1}^{n} q_{j} \in \bar{V}^{-}\right\} .
$$

We let $\bar{V}_{\varepsilon}^{+}$denote the $\varepsilon$-neighborhood of the closed forward light cone $\bar{V}^{+}$and $\chi_{\varepsilon}$ denote a smoothed characteristic function of $\bar{V}^{+}$with the properties: $\chi_{\varepsilon}$ is identically 1 on

\footnotetext{
${ }^{2}$ As usual, we assume that the type of commutation relation is the same for all components of a field.
} 
$\bar{V}_{\varepsilon / 2}^{+}$, vanishes outside $\bar{V}_{\varepsilon}^{+}$and is a multiplier of $S\left(\mathbb{R}^{4}\right)$. Because $(u, f)=(2 \pi)^{-d}(\hat{u}, \hat{f}(-\cdot))$ for any $u \in S^{\prime}\left(\mathbb{R}^{d}\right)$ and $f \in S\left(\mathbb{R}^{d}\right)$, it follows from (25) that the matrix element (24) is unchanged on replacing $h$ and $g$ with functions $h_{\varepsilon}$ and $g_{\varepsilon}$ such that $\widehat{h_{\varepsilon}^{*}}\left(k_{1}, \ldots, k_{m}\right)=$ $\chi_{\varepsilon}\left(-\sum_{j} k_{j}\right) \widehat{h^{*}}\left(k_{1}, \ldots, k_{m}\right)$ and $\hat{g}_{\varepsilon}\left(q_{1}, \ldots, q_{n}\right)=\chi_{\varepsilon}\left(\sum_{j} q_{j}\right) \hat{g}\left(q_{1}, \ldots, q_{n}\right)$. From (19) we have

$$
\operatorname{supp}\left(h_{\varepsilon}^{*} \otimes_{\theta} f_{1}\right) \otimes\left(f_{2} \otimes_{-\theta} g_{\varepsilon}\right) \subset \mathbb{R}^{4 m} \times\left(\operatorname{supp} f_{1}+\frac{1}{2} \theta \bar{V}_{\varepsilon}^{-}\right) \times\left(\operatorname{supp} f_{2}+\frac{1}{2} \theta \bar{V}_{\varepsilon}^{+}\right) \times \mathbb{R}^{4 n} .
$$

The inclusion $\theta_{1} V^{-} \subset \mathrm{W}_{1}$ implies that $\frac{1}{2} \theta V^{-} \subset \mathrm{W}_{\theta}$ and $\frac{1}{2} \theta V^{+} \subset-\mathrm{W}_{\theta}$. Therefore, if the supports of $f_{1}$ and $f_{2}$ are compact and contained, respectively, in $\mathrm{W}_{\theta}$ and $-\mathrm{W}_{\theta}$, and if $\varepsilon$ is sufficiently small, then supp $f_{1}+\frac{1}{2} \theta \bar{V}_{\varepsilon}^{-} \subset \mathrm{W}_{\theta}$ and $\operatorname{supp} f_{2}+\frac{1}{2} \theta \bar{V}_{\varepsilon}^{+} \subset-\mathrm{W}_{\theta}$. Because $\left(x_{1}-x_{2}\right)^{2}<0$ for any $x_{1} \in \mathrm{W}_{\theta}$ and $x_{2} \in-\mathrm{W}_{\theta}$, we conclude that the equality (23) follows from the locality of the undeformed fields which means, in terms of the Wightman functions, that $w-w_{\pi}$ vanishes for $\left(x_{1}-x_{2}\right)^{2}<0$. For the case of anticommuting fields, the reasoning is the same but with obvious changes of signs.

Because the deformation preserves the translation invariance, (22) also clearly holds if there exists a translation $a$ such that

$$
\operatorname{supp} f_{1}+a \subset \mathrm{W}_{\theta}, \quad \operatorname{supp} f_{2}+a \subset-\mathrm{W}_{\theta} .
$$

Remark 1 . The derivation of Theorem 4.5 in [15] relies on the assertion that the Fourier transform of the distribution defined by $\left\langle\Psi,\left[\phi_{\iota}^{\theta}\left(f_{1}\right), \phi_{\iota^{\prime}}^{-\theta}\left(f_{2}\right)\right] \Phi_{\iota_{1} \ldots \iota_{n}}(g)\right\rangle$, where $\Psi \in \mathcal{H}$, has support in the $(n+2)$-fold product of the forward light cone $\bar{V}^{+}$. This contradicts (22) because then this distribution would be the boundary value of an analytic function and hence could not vanish identically on a non-empty open set. Nevertheless, as shown above, the theorem's conclusion holds. Another proof of the wedge-local (anti)commutation relations for the deformed fields is given by Lechner [18] in an operator-algebraic setting.

\section{ASYMPTOTIC COMMUTATIVITY}

Theorem 1 says that the field (anti)commutator $\left[\phi_{\iota}^{\theta}\left(x_{1}\right), \phi_{\iota^{\prime}}^{-\theta}\left(x_{2}\right)\right]_{(+)}$vanishes identically on $\mathrm{W}_{\theta} \times \mathrm{W}_{-\theta}$. In this section, we show that it also has a rapid decrease (in the sense of generalized functions) in the whole spacelike region $\left(x_{1}-x_{2}\right)^{2}<0$. As before, we restrict our consideration to the case of commutator. Let $\Psi$ be an arbitrary vector in $\mathcal{H}$, let $\Phi$ belong to $D$, and let

$$
u_{\Psi, \Phi}\left(f_{1}, f_{2}\right)=\left\langle\Psi,\left[\phi_{\iota}^{\theta}\left(f_{1}\right), \phi_{\iota^{\prime}}^{-\theta}\left(f_{2}\right)\right] \Phi\right\rangle .
$$


By the Schwartz kernel theorem, the bilinear functional (26) is identified with a distribution in $S^{\prime}\left(\mathbb{R}^{4} \times \mathbb{R}^{4}\right)$. A simple way of describing the behavior of a distribution at infinity is by examining its convolution with test functions decreasing sufficiently fast. Therefore, we should consider the asymptotic behavior of the convolution $u_{\Psi, \Phi} * f$ with adequately chosen functions $f$. This convolution may be written symbolically in the form

$$
\left(u_{\Psi, \Phi} * f\right)\left(x_{1}, x_{2}\right)=\int\left\langle\Psi,\left[\phi_{\iota}^{\theta}\left(\xi_{1}\right), \phi_{\iota^{\prime}}^{-\theta}\left(\xi_{2}\right)\right] \Phi\right\rangle f\left(x_{1}-\xi_{1}, x_{2}-\xi_{2}\right) d \xi_{1} d \xi_{2} .
$$

We will use the Gelfand-Shilov test function spaces $S_{\alpha}^{\beta}$ which are contained in $S$. If $\beta<1$, the definition of these spaces can be formulated in terms of complex variables, which considerably simplifies the estimates of Theorem [2 below. As shown in [30], the elements of $S_{\alpha}^{\beta}\left(\mathbb{R}^{d}\right)$, where $\beta<1$, can be continued analytically into $\mathbb{C}^{d}$, and $S_{\alpha}^{\beta}$ is isomorphic to the space of entire functions $W_{\rho}^{\sigma}$, where $\rho=1 / \alpha$ and $\sigma=1 /(1-\beta)>1$. The functions belonging to $W_{\rho}^{\sigma}$ satisfy the inequality

$$
|f(x+i y)| \leq C \prod_{j=1}^{d} e^{-a\left|x_{j}\right|^{\rho}+b\left|y_{j}\right|^{\sigma}}
$$

with some positive constants $a, b$, and $C$ depending on $f$. The norm corresponding to (28) is given by

$$
\|f\|_{a, b}=\sup _{z=x+i y}|f(z)| \prod_{j=1}^{d} e^{a\left|x_{j}\right|^{\rho}-b\left|y_{j}\right|^{\sigma}}
$$

and we let $W_{\rho, a}^{\sigma, b}$ denote the space of entire functions such that $\|f\|_{\bar{a}, \bar{b}}<\infty$ for all positive $\bar{a}<a$ and $\bar{b}>b$. Clearly,

$$
W_{\gamma}^{\rho}=\bigcup_{a \rightarrow 0, b \rightarrow \infty} W_{\gamma, a}^{\rho, b}
$$

If $\sigma>\rho$, then the space $W_{\rho, a}^{\sigma, b}$ is nontrivial for any $a>0$ and $b>0$, but if $\sigma=\rho, W_{\rho, a}^{\sigma, b}$ is nontrivial only under the condition $a \geq b$. Indeed, if $a<b$, then (28) implies that $f(z) \cdot f(i z)$ tends to zero as $|z| \rightarrow \infty$ and is hence identically zero by the Liouville theorem. The same argument shows that $W_{\rho}^{\sigma}$ is trivial for $\sigma<\rho$. Under the condition $\rho>1$, the Fourier transformation is an isomorphism of $W_{\rho, a}^{\sigma, b}$ onto $W_{\sigma^{\prime}, b^{\prime}}^{\rho^{\prime}, a^{\prime}}$, where the primed indices are defined by the duality relations

$$
\frac{1}{\rho^{\prime}}+\frac{1}{\rho}=1, \quad\left(\rho^{\prime} a^{\prime}\right)^{\rho}(\rho a)^{\rho^{\prime}}=1
$$

and by analogous relations for $\sigma^{\prime}, b^{\prime}$. We will also use the spaces $W^{\sigma, b}$ defined by

$$
|f(z)| \leq C_{N, \bar{b}}(1+|x|)^{-N} \prod_{j=1}^{d} e^{\bar{b}\left|y_{j}\right|^{\sigma}}, \quad \bar{b}>b, \quad N=0,1,2 \ldots,
$$


and the spaces $W^{\sigma}=\bigcup_{b \rightarrow \infty} W^{\sigma, b}$, which are isomorphic to the Gelfand-Shilov spaces $S^{\beta}$ with $\beta=1-1 / \sigma$. The Fourier transformation maps $W^{\sigma}$ onto the space $W_{\sigma^{\prime}}=S_{1 / \sigma^{\prime}}$ and $W^{\sigma, b}$ onto the space $W_{\sigma^{\prime}, b^{\prime}}$ of smooth functions on $\mathbb{R}^{d}$ with the norms

$$
\|g\|_{N, \bar{b}^{\prime}}=\max _{|\kappa|<N} \sup _{p} \prod_{j=1}^{d} e^{\bar{b}^{\prime}\left|p_{j}\right| \sigma^{\prime}}\left|\partial^{\kappa} g(p)\right|, \quad \bar{b}^{\prime}<b^{\prime}, \quad N=0,1,2, \ldots
$$

The choice of norm $|\cdot|$ on $\mathbb{R}^{d}$ is inessential to the definition (31), but when working with functions in $W_{\rho}^{\sigma}$, it is convenient to use the norm

$$
|x|=\left(\sum_{j}\left|x_{j}\right|^{\rho}\right)^{1 / \rho}
$$

We need two auxiliary lemmas.

Lemma 1 . Let $u$ be a distribution on $\mathbb{R}^{d}$ with support in a closed cone $V \neq \mathbb{R}^{d}$, and let $G$ be a closed cone such that $G \cap V=\{0\}$. If $f \in W_{\rho, a}$, then for some $N$ and for any $\overline{\bar{a}}<\bar{a}<a$, the convolution $(u * f)(x)$ satisfies the estimate

$$
|(u * f)(x)| \leq C_{G, \bar{a}, \overline{\bar{a}}}\|f\|_{N, \bar{a}} e^{-\overline{\bar{a}}\left|d_{G, V} x\right|^{\rho}}, \quad x \in G,
$$

where the norm $|\cdot|$ on $\mathbb{R}^{d}$ is given by (32) and $d_{G, V}=\inf _{x \in G,|x|=1} \inf _{\xi \in V}|x-\xi|$.

Proof. For simplicity we assume that the set $V$ is regular. ${ }^{3}$ Then there exist a constant $C>0$ and a integer $N$ (both depending on $u$ ) such that

$$
|(u, f)| \leq C \max _{|\kappa| \leq N} \sup _{\xi \in V}(1+|\xi|)^{N}\left|\partial^{\kappa} f(\xi)\right| \text { for all } f \in S\left(\mathbb{R}^{d}\right) .
$$

If the regularity condition is not satisfied, then $V$ in (34) should be replaced by its $\varepsilon$ neighborhood. This slightly complicates the analysis, but does not change the result. By the definition of norms in $W_{\rho, a}$, we have $\left|\partial^{\kappa} f(\xi)\right| \leq\|f\|_{|\kappa|, \bar{a}} e^{-\bar{a}|\xi|^{\rho}}$ for any $\kappa$ and $\bar{a}<a$. Replacing the function $f(\xi)$ by $f(x-\xi)$ and using (34), we obtain

$$
\begin{gathered}
|(u * f)(x)| \leq C\|f\|_{N, \bar{a}} \sup _{\xi \in V}(1+|\xi|)^{N} e^{-\bar{a}|x-\xi|^{\rho}} \leq \\
\leq C\|f\|_{N, \bar{a}}(1+|x|)^{N} \sup _{\xi \in V}(1+|x-\xi|)^{N} e^{-\bar{a}|x-\xi|^{\rho}} \leq \\
\quad \leq C_{\bar{a}_{1}}\|f\|_{N, \bar{a}}(1+|x|)^{N} e^{-\bar{a}_{1}\left|d_{V}(x)\right|^{\rho}}
\end{gathered}
$$

\footnotetext{
${ }^{3}$ See definition in 29], Supplement A.2. The closed light cone is a regular set.
} 
where $\bar{a}_{1}<\bar{a}$ and can be chosen arbitrarily close to $\bar{a}$, and $d_{V}(x)=\inf _{\xi \in V}|x-\xi|$. Because the cone $V$ is invariant under dilations, we have

$$
d_{V}(x)=|x| \inf _{\xi \in V}|x /| x|-\xi|=|x| d_{V}(x /|x|) .
$$

It follows from the condition $G \cap V=\{0\}$ that $d_{G, V}=\inf _{x \in G,|x|=1} d_{V}(x)>0$. Therefore the factor $(1+|x|)^{N}$ can be omitted from the last row in (35), slightly decreasing $\bar{a}_{1}$, and we arrive at (33). Lemma 1 is proved.

Remark 2 . We consider below a special case, where $d=d_{1}+d_{2}$ and $\operatorname{supp} u \subset \mathbb{V} \times \mathbb{R}^{d_{2}}$, with $\mathbb{V}$ a cone in $\mathbb{R}^{d_{1}}$. Then an estimate analogous to (33) holds in any closed cone $\mathbb{G} \subset \mathbb{R}^{d_{1}}$ such that $\mathbb{G} \cap \mathbb{V}=\{0\}$ and even under a weaker assumption on the behavior of $f$ with respect to the second group of variables. In particular, those functions are admissible that satisfy the conditions

$$
\max _{|\kappa| \leq N}\left|\partial^{\kappa} f\left(x, x^{\prime}\right)\right| \leq C_{f, N, \bar{a}} e^{-\bar{a}|x|^{\rho}}\left(1+\left|x^{\prime}\right|\right)^{-N}, \quad N=0,1,2, \ldots
$$

From (36), we obtain an estimate of type (33) for $(u * f)(x, 0)$, but with $C_{f, N, \bar{a}}$ in place of $\|f\|_{N, \bar{a}}$. The function space defined by (36) is the completed tensor product $W_{\rho, a}\left(\mathbb{R}^{d_{1}}\right) \hat{\otimes} S\left(\mathbb{R}^{d_{2}}\right)$.

Lemma 2 . If $\sigma^{\prime}>\rho=\rho^{\prime} /\left(\rho^{\prime}-1\right)$, then for every quadratic form $\mathcal{Q}(p)$ with real coefficients, the function $e^{i \mathcal{Q}(p)}$ is a multiplier of $W_{\sigma^{\prime}, b}^{\rho^{\prime}, a}$ for any $a>0, b>0$.

Proof. We need to estimate the function $\left|e^{i \mathcal{Q}(p+i s)}\right|=e^{-\operatorname{Im} \mathcal{Q}(p+i s)}$. Let $\mathcal{Q}_{j k}$ be the matrix of the quadratic form $\mathcal{Q}$ and let $|\mathcal{Q}|=\max _{j, k}\left|Q_{j k}\right|$. Young's inequality for products states that if $r$ and $t$ are nonnegative real numbers and $\rho$ and $\rho^{\prime}$ are positive numbers satisfying the first of duality relations (30), then $r t \leq r^{\rho} / \rho+t^{\rho^{\prime}} / \rho^{\prime}$. Using this inequality with $r=\left|p_{j}\right| / \varepsilon$ and $t=\varepsilon\left|s_{k}\right|$, where $\varepsilon>0$, we obtain

$$
\begin{aligned}
\left|\operatorname{Im} \sum_{j, k}\left(p_{j}+i s_{j}\right) \mathcal{Q}_{j k}\left(p_{k}+i s_{k}\right)\right| \leq 2|\mathcal{Q}| \sum_{j . k}\left|p_{j} s_{k}\right| & \leq \\
& \leq 2 d|\mathcal{Q}| \sum_{j}\left(\frac{1}{\rho}\left|\frac{p_{j}}{\varepsilon}\right|^{\rho}+\frac{1}{\rho^{\prime}}\left|\varepsilon s_{j}\right|^{\rho^{\prime}}\right) .
\end{aligned}
$$

The condition $\rho<\sigma^{\prime}$ implies that for arbitrarily small $\varepsilon$, there is a constant $C_{\varepsilon}>0$ such that

$$
\left|p_{j} / \varepsilon\right|^{\rho} \leq C_{\varepsilon}+\varepsilon\left|p_{j}\right|^{\sigma^{\prime}}
$$


In line with (29), the norms in $W_{\sigma^{\prime}}^{\rho^{\prime}}$ are defined by $\|g\|_{a, b}=\sup _{p, s}|g(p+i s)| \prod_{j=1}^{d} e^{a\left|p_{j}\right|^{\sigma^{\prime}}-b\left|s_{j}\right|^{\rho^{\prime}}}$. Substituting (38) into (37), we conclude that for any $\bar{a}_{1}<\bar{a}<a$ and $\bar{b}_{1}>\bar{b}>b$, there exists a constant $C_{\bar{a}_{1}, \bar{b}_{1}}$ such that

$$
\left\|g e^{i \mathcal{Q}}\right\|_{\bar{a}_{1}, \bar{b}_{1}} \leq C_{\bar{a}_{1}, \bar{b}_{1}}\|g\|_{\bar{a}, \bar{b}} \quad \text { for all } g \in W_{\sigma^{\prime}, b}^{\rho^{\prime}, a}
$$

which completes the proof.

Remark 3 . If $\sigma^{\prime}=\rho$, then $e^{i Q(p)}$ is a multiplier of $W_{\sigma^{\prime}}^{\rho^{\prime}}$, but not of $W_{\sigma^{\prime}, b}^{\rho^{\prime}, a}$. The condition $\sigma^{\prime} \geq \rho$ for the spaces of type $W$ is equivalent to the condition $\alpha \geq \beta$ for $S_{\alpha}^{\beta}$. As shown in [25], only under this condition $S_{\alpha}^{\beta}$ is an algebra with respect to the Weyl-Moyal product (4).

We now turn to describing the asymptotic behavior of distribution (26) at large spacelike separations. The corresponding theorem is accompanied below by a simple but explanatory example. We let $\mathbb{V}$ denote the cone in $\mathbb{R}^{4 \cdot 2}=\mathbb{R}^{4} \times \mathbb{R}^{4}$ consisting of the pairs $\left(x_{1}, x_{2}\right)$ such that $x_{1}-x_{2}$ belongs to the closed light cone,

$$
\mathbb{V}=\left\{\left(x_{1}, x_{2}\right) \in \mathbb{R}^{4 \cdot 2}:\left(x_{1}-x_{2}\right)^{2} \geq 0\right\}
$$

Theorem 2 . Let $\Phi(g)$ be a vector of the form (13) with $g \in W^{\sigma}\left(\mathbb{R}^{4 n}\right)$, let $\Psi$ be an arbitrary vector in $\mathcal{H}$, and let $\mathbb{G}$ be a closed cone in $\mathbb{R}^{4 \cdot 2}$ such that $\mathbb{G} \cap \mathbb{V}=\{0\}$. If $f \in W_{\rho}^{\sigma}\left(\mathbb{R}^{4 \cdot 2}\right)$, where $\rho<\sigma^{\prime}=\sigma /(\sigma-1)$ and $\|f\|_{a, b}<\infty$, then for any $\bar{a}<a$, the function (27) satisfies the estimate

$$
\left|\left(u_{\Psi, \Phi} * f\right)\left(x_{1}, x_{2}\right)\right| \leq C_{G, \bar{a} \Psi, \Phi}\|f\|_{a, b} \exp \left\{-\bar{a} d_{\mathbb{G}, \mathbb{V}}^{\rho}\left(\left|x_{1}\right|^{\rho}+\left|x_{2}\right|^{\rho}\right)\right\}, \quad\left(x_{1}, x_{2}\right) \in \mathbb{G}
$$

where the angular distance $d_{\mathbb{G}, \mathbb{V}}$ is defined in Lemma 1 .

Proof. Let $u_{\Psi}$ be the distribution defined on $\mathbb{R}^{4(2+n)}$ by the three-linear functional $\left\langle\Psi,\left[\phi_{\iota}\left(f_{1}\right), \phi_{\iota^{\prime}}\left(f_{2}\right)\right] \Phi(g)\right\rangle$. By locality of the undeformed fields, its support lies in the cone $\mathbb{V} \times \mathbb{R}^{4 n}$, and (16) implies that

$$
\left(u_{\Psi, \Phi}, f_{1} \otimes f_{2}\right)=\left(u_{\Psi}, f_{1} \otimes_{\theta}\left(f_{2} \otimes_{-\theta} g\right)\right) \quad \text { for all } f_{1}, f_{2} \in S\left(\mathbb{R}^{4}\right) \text { and } g \in S\left(\mathbb{R}^{4 n}\right) .
$$

By the definition of the product $\otimes_{\theta}$, the Fourier transform of $f_{1} \otimes_{\theta}\left(f_{2} \otimes_{-\theta} g\right)$ has the form $\eta \cdot\left(\hat{f}_{1} \otimes \hat{f}_{2} \otimes \hat{g}\right)$, where

$$
\eta\left(p_{1}, p_{2}, q\right)=e^{-\frac{i}{2}\left(p_{1} \theta p_{2}+\left(p_{1}-p_{2}\right) \theta \sum_{j=1}^{n} q_{j}\right)} .
$$


Since $S\left(\mathbb{R}^{4}\right) \otimes S\left(\mathbb{R}^{4}\right)$ is dense in $S\left(\mathbb{R}^{4 \cdot 2}\right)$, it follows from (40) that for any $f \in S\left(\mathbb{R}^{4 \cdot 2}\right)$,

$$
\left(u_{\Psi, \Phi}, f\right)=\left(u_{\Psi}, h_{f}\right), \quad \text { where } \hat{h}_{f} \stackrel{\text { def }}{=} \eta \cdot(\hat{f} \otimes \hat{g}) \in S\left(\mathbb{R}^{4(2+n)}\right) .
$$

Therefore,

$$
\left(u_{\Psi, \Phi} * f\right)\left(x_{1}, x_{2}\right)=\left(u_{\Psi} * h_{f}\right)\left(x_{1}, x_{2}, 0\right) .
$$

If $f \in W_{\rho}^{\sigma}\left(\mathbb{R}^{4 \cdot 2}\right)$ and $g \in W_{\rho}^{\sigma}\left(\mathbb{R}^{4 n}\right)$, then $\|f \otimes g\|_{a, b}=\|f\|_{a, b}\|g\|_{a, b}$ by the definition (29). The Fourier transformation, as already said, is an isomorphism of $W_{\rho, a}^{\sigma, b}$ onto $W_{\sigma^{\prime}, b^{\prime}}^{\rho^{\prime}, a^{\prime}}$, and by Lemma 2 the function $\eta$ is a multiplier of $W_{\sigma^{\prime}, b^{\prime}}^{\rho^{\prime}, a^{\prime}}\left(\mathbb{R}^{4(n+2)}\right)$ under the condition $\rho<\sigma^{\prime}$. Hence the correspondence $f \rightarrow h_{f}$ is continuous from $W_{\rho, a}^{\sigma, b}\left(\mathbb{R}^{4 \cdot 2}\right)$ to $W_{\rho, a}^{\sigma, b}\left(\mathbb{R}^{4(n+2)}\right)$, and for any $\bar{a}_{1}<\bar{a}<a$ and $\bar{b}_{1}>\bar{b}>b$, we have

$$
\left\|h_{f}\right\|_{\bar{a}_{1}, \bar{b}_{1}} \leq C_{\bar{a}_{1}, \bar{b}_{1}}\|f\|_{\bar{a}, \bar{b}} \leq C_{\bar{a}_{1}, \bar{b}_{1}}\|f\|_{a, b}
$$

The operation of differentiation is continuous in $W_{\rho, a}^{\sigma, b}$, as can easily be seen by using Cauchy's formula. Therefore, $W_{\rho, a}$ is continuously embedded in $W_{\rho, a}^{\sigma, b}$, and

$$
\left\|h_{f}\right\|_{N, \bar{a}_{2}} \leq C_{\kappa, \bar{a}_{2}}\left\|h_{f}\right\|_{\bar{a}_{1}, \bar{b}_{1}} \text { for any } N \text { and } \bar{a}_{2}<\bar{a}_{1} \text {. }
$$

Applying Lemma 1 to the right-hand side of (42) and using (43) and (44), we arrive at (39). Now let $g \in W^{\sigma, B}$. Performing the Fourier transformation, using the condition $\rho<\sigma^{\prime}$, and making the inverse transformation, we obtain

$$
\left|h_{f}\left(x+i y, x^{\prime}+i y^{\prime}\right)\right| \leq C_{g, \bar{a}, \bar{b}, \bar{B}}\|f\|_{a, b} e^{-\bar{a}|x|^{\rho}}\left(1+\left|x^{\prime}\right|\right)^{-N} \prod_{j, \mu} e^{\bar{b}\left|y_{j}^{\mu}\right|^{\sigma}+\bar{B}\left|y_{j}^{\prime \mu}\right|^{\sigma}} .
$$

where $x$ denotes the pair $\left(x_{1}, x_{2}\right)$ and $x^{\prime}$ denotes the variables of $g$. Hence $h_{f}\left(x, x^{\prime}\right)$ satisfies inequalities of type (36) with a constant $C_{f, N, \bar{a}}$ proportional to $\|f\|_{a, b}$. Invoking Remark 2 , we arrive again at (39), which completes the proof.

Remark 4 . The condition $\mathbb{G} \cap \mathbb{V}=\{0\}$ implies that $\mathbb{G}$ is contained in a wedge of the form $\left\{\left(x_{1}, x_{2}\right): x_{1}-x_{2} \in G\right\}$, where $G$ is a closed cone in $\mathbb{R}^{4}$ having only the origin in common with the closed light cone $\bar{V}$. The norm (32) dominates the Euclidean norm $\|\cdot\|$ and using the parallelogram identity, we see that for all $x_{1}-x_{2} \in G$, the following inequality holds:

$$
\left|\left(u_{\Psi, \Phi} * f\right)\left(x_{1}, x_{2}\right)\right| \leq C_{G, \Psi, \Phi}\|f\|_{a, b} \exp \left\{-\frac{a}{2} \delta_{G, \bar{V}}\left\|x_{1}-x_{2}\right\|^{\rho}\right\}
$$

where the distance $\delta_{G, \bar{V}}$ is defined by the Euclidean norm. 
As an explanatory example, consider the matrix element

$$
u_{g_{1}, g_{2}}\left(f_{1}, f_{2}\right)=\left\langle\varphi\left(g_{1}\right) \Omega,\left[\varphi^{\theta}\left(f_{1}\right), \varphi^{-\theta}\left(f_{2}\right)\right] \varphi\left(g_{2}\right) \Omega\right\rangle,
$$

where $\varphi$ is a free massive neutral scalar field. Expressing the four-point vacuum expectation value in terms of two-point ones and passing to the momentum representation, (45) can be written as

$$
\begin{array}{r}
u_{g_{1}, g_{2}}\left(f_{1}, f_{2}\right)=\frac{i}{(2 \pi)^{8}} \int d k d p_{1} d p_{2} d q \delta(k+q) \delta\left(p_{1}+p_{2}\right) \hat{\Delta}_{+}(k) \hat{\Delta}\left(p_{1}\right) \\
\times e^{-\frac{i}{2}\left(p_{1} \theta p_{2}+p_{1} \theta q-p_{2} \theta q\right)} \overline{\hat{g}_{1}(k)} \hat{f}_{1}\left(-p_{1}\right) \hat{f}_{2}\left(-p_{2}\right) \hat{g}_{2}(-q) \\
=\frac{i}{(2 \pi)^{8}} \int d k d p \hat{\Delta}_{+}(k) \hat{\Delta}(p) e^{i p \theta k} \overline{\hat{g}_{1}(k)} \hat{f}_{1}(-p) \hat{f}_{2}(p) \hat{g}_{2}(k),
\end{array}
$$

where $\hat{\Delta}(p)=-2 \pi i \epsilon\left(p^{0}\right) \delta\left(p^{2}-m^{2}\right)$ is the Fourier transform of the Pauli-Jordan function, and $\hat{\Delta}_{+}$is its positive-frequency part. Letting $\hat{f}(p)=\hat{f}_{1}(p) \hat{f}_{2}(-p)$ and $\hat{g}(k)=\overline{\hat{g}_{1}(-k)} \hat{g}_{2}(-k)$ and turning back to the coordinate representation, we obtain

$$
u_{g_{1}, g_{2}}\left(f_{1}, f_{2}\right)=i\left(\Delta_{+} \otimes_{2 \theta} \Delta, g \otimes f\right)=i\left(\Delta_{+} \otimes \Delta, g \otimes_{2 \theta} f\right) .
$$

If $\operatorname{supp} f_{1} \subset \mathrm{W}_{\theta}$ and $\operatorname{supp} f_{2} \subset \mathrm{W}_{-\theta}$, then $f$, being the convolution product of $f_{1}(\xi)$ and $f_{2}(-\xi)$, is supported in $\mathrm{W}_{\theta}$. Taking into consideration the support properties of $\hat{\Delta}_{+}$, we conclude, as in Sec. III, that $\operatorname{supp}\left(g \otimes_{2 \theta} f\right) \subset \mathrm{W}_{\theta}+\theta V^{-} \subset \mathrm{W}_{\theta}$, and hence $u_{g_{1}, g_{2}}\left(f_{1}, f_{2}\right)$ vanishes for such test functions. To test the behavior of (45) for arbitrary spacelike separations, we use the shifted test functions $f_{1}\left(x_{1}-\xi\right)$ and $f_{2}\left(x_{2}-\xi\right)$, where $\left(x_{1}-x_{2}\right)^{2}<0$. Then $f(\xi)$ is replaced by $f\left(x_{1}-x_{2}-\xi\right)$ and $g \otimes_{2 \theta} f$ is shifted away from the support of $\Delta_{+} \otimes \Delta$. Therefore, $u_{g_{1}, g_{2}}\left(f_{1}\left(x_{1}-\cdot\right), f_{2}\left(x_{2}-\cdot\right)\right)$ inherits the fall-off properties of $g \otimes_{2 \theta} f$. It is clear from (18) that the rate of decrease of $g \otimes_{2 \theta} f$ in the $x$-variable is the same as that of $f$, if the latter decreases slower than $\hat{g}$ does. In technical terms, if $\hat{g}_{2}(k)$, and hence $\hat{g}(k)$, belongs to $W_{\sigma^{\prime}}=\widehat{W}^{\sigma}$ and falls off as $e^{-|k|^{\sigma^{\prime}}}$, we take $f_{1,2}$ in $W_{\rho}$ with $\rho<\sigma^{\prime}$ and conclude that $u_{g_{1}, g_{2}}\left(f_{1}\left(x_{1}-\cdot\right), f_{2}\left(x_{2}-\cdot\right)\right)$ decreases with a rate characterized by Lemma 1 . In the case of interacting fields, the occurrence of the term $-\frac{i}{2} p_{1} \theta p_{2}$ in (41) forces us to take $f_{1}$ and $f_{2}$ in $W_{\rho} \cap W^{\sigma}=W_{\rho}^{\sigma}$. With such a choice, the function (27) falls off in the same manner in all spacelike directions.

It should be pointed out that the linear subspace spanned by the vacuum $\Omega$ and all vectors of the form (13) with $g \in W^{\sigma}$ is dense in $\mathcal{H}$, because $W^{\sigma}$ is dense in $S$. Any $W^{\sigma}$ contains $W^{1}$ whose Fourier transform is nothing but the space $C_{0}^{\infty}$ of all infinitely differentiable functions 
of compact support, and $C_{0}^{\infty}$ is just the function space that is employed in the Haag-Ruelle scattering theory. The condition $\rho<\sigma^{\prime}$, together with the condition $\rho \leq \sigma$ of non-triviality of $W_{\rho}^{\sigma}$, implies that $\rho<2$, because $\min \left(\sigma, \sigma^{\prime}\right) \leq 2$. Since $\rho$ can be chosen arbitrarily close to 2 , Theorem 2 merely says that the commutator $\left[\phi_{\iota}^{\theta}\left(x_{1}\right), \phi_{\iota^{\prime}}^{-\theta}\left(x_{2}\right)\right]$ decreases approximately as a Gaussian at large spacelike separation of $x_{1}$ and $x_{2}$. The borderline case $\rho=\sigma$, i.e., the case of test functions in $W_{\sigma}^{\sigma}=S_{1-\beta}^{\beta}$, where $\beta=1-1 / \sigma=1 / \sigma^{\prime}$, is of particular interest. ${ }^{4}$ Theorem 3 of [26] shows, that in this case, (39) amounts to the condition that the distribution $u_{\Psi, \Phi}$ has a continuous extension to the space $S^{\beta}(\mathbb{V})=W^{\sigma}(\mathbb{V})$ of entire functions satisfying the inequalities

$$
|f(x+i y)| \leq C_{N}(1+|x|)^{-N} \exp \left\{b d_{U}^{\sigma}(x)+b|y|^{\sigma}\right\}, \quad N=0,1,2, \ldots
$$

where $U$ is an open cone, depending on $f$, such that $\mathbb{V} \backslash\{0\} \subset U$, and where $C_{N}$ and $b$ are positive constants, also depending on $f$. Conditions of this kind were earlier used in nonlocal quantum field theory, where the framework of tempered distributions appears to be too restrictive and the adequate choice of test function space takes on great significance. In particular, the spaces $S^{\beta}(\mathbb{V})$ with $\beta<1$, consisting of analytic functions, were used in formulating an asymptotic commutativity principle replacing local commutativity for nonlocal fields. In [26], nonlocal fields $\phi_{\iota}$ and $\phi_{\iota^{\prime}}$ defined as operator-valued generalized functions on $S^{\beta}\left(\mathbb{R}^{4}\right)$ are referred to as asymptotically (anti)commuting, if the matrix element $\left\langle\Psi,\left[\phi_{\iota}\left(x_{1}\right), \phi_{\iota^{\prime}}\left(x_{2}\right)\right]_{(+)} \Phi\right\rangle$ has a continuous extension to $S^{\beta}(\mathbb{V})$ for any vectors $\Phi$ and $\Psi$ in their common dense domain in the Hilbert space. The principle of asymptotic commutativity implies that any two field components either commute or anticommute asymptotically at large spacelike separation of the arguments. This condition provides a way of extending the CPT and spin-statistics theorems to nonlocal QFT [24]. The condition of mutual asymptotic commutativity was also used in [31] to extend the Borchers equivalence classes to nonlocal fields. Theorem 2 shows that the deformed fields $\phi_{\iota}^{\theta}$ and $\phi_{\iota^{\prime}}^{-\theta}$ (more precisely, their restrictions to the test functions in $S^{\beta}\left(\mathbb{R}^{4}\right), \beta<1 / 2$ ) (anti)commute asymptotically if the initial fields $\phi_{\iota}$ and $\phi_{\iota^{\prime}}$ (anti)commute at spacelike separation. Using the fact that the twist operator (3) is an automorphism of $S^{\beta}(\mathbb{V})$ for $\beta<1 / 2$, an extension of the distribution $\left\langle\Psi,\left[\phi_{\iota}^{\theta}\left(x_{1}\right), \phi_{\iota^{\prime}}^{-\theta}\left(x_{2}\right)\right]_{(+)} \Phi\right\rangle$ to this space can explicitly be constructed, but here we find it preferable to define the asymptotic commutativity as a fall-off property of the smoothed field commutator, which clearly shows its meaning.

\footnotetext{
${ }^{4}$ We note that $\rho<\sigma^{\prime}$ implies $\beta<1 / 2$.
} 


\section{CLUSTER PROPERTIES OF THE DEFORMED WIGHTMAN FUNCTIONS}

In order to prove the uniqueness of the vacuum state in reconstructing quantum fields from a given set of Wightman functions, it suffices to use the cluster decomposition property

$$
w_{\boldsymbol{\iota}_{1} \ldots \iota_{n}}\left(f \otimes g_{(\lambda a)}\right) \longrightarrow w_{\iota_{1} \ldots \iota_{m}}(f) w_{\boldsymbol{\iota}_{m+1} \ldots \iota_{n}}(g) \quad(\lambda \rightarrow \infty),
$$

where $f \in S\left(\mathbb{R}^{4 m}\right), g \in S\left(\mathbb{R}^{4(n-m)}\right)$, and $a$ is an arbitrary spacelike vector. However, Theorem 3-4 of [16] shows that the vacuum expectation values of local field theory satisfy the slightly stronger condition

$$
w_{\iota_{1} \ldots \iota_{n}}\left(h_{(m, \lambda a)}\right) \longrightarrow\left(w_{\iota_{1} \ldots \iota_{m}} \otimes w_{\iota_{m+1} \ldots \iota_{n}}\right)(h) \quad(\lambda \rightarrow \infty),
$$

where $h$ is any function in $S\left(\mathbb{R}^{4 n}\right)$ and

$$
h_{(m, \lambda a)}\left(x_{1}, \ldots, x_{n}\right)=h\left(x_{1}, \ldots, x_{m}, x_{m+1}-\lambda a, \ldots, x_{n}-\lambda a\right) .
$$

Setting

$$
\hat{h}=\prod_{1 \leq j<k \leq n} e^{-\frac{i}{2} p_{j} \theta p_{k}}(\hat{f} \otimes \hat{g})
$$

and using (8) and (11), we see that the limit relation (48) implies (12) for the deformed Wightman functions, because the distribution $w_{\iota_{1} \ldots \iota_{m}} \otimes w_{\iota_{m+1} \ldots \iota_{n}}$ contains the factor $\delta\left(\sum_{j=1}^{m} p_{j}\right) \delta\left(\sum_{j=m+1}^{n} p_{j}\right)$ by the translation invariance.

The Haag-Ruelle scattering theory uses essentially the decomposition of vacuum expectation values into truncated ones. The truncated Wightman functions $w^{T}$ are obtained by eliminating the contribution of the intermediate vacuum state from the support of $\hat{w}$, see [29, 32]. If zero is an isolated point of the spectrum of the energy-momentum operator, i.e., the spectrum has the form

$$
\text { sp } P \subset\{0\} \cup \bar{V}_{\mu}^{+}, \quad \text { where } \bar{V}_{\mu}^{+}=\left\{p: p_{0} \geq \sqrt{\mathbf{p}^{2}+\mu^{2}}\right\} \text { and } \mu>0 \text {, }
$$

then supp $\hat{w}^{T}\left(p_{1}, \ldots, p_{n}\right)$ is contained in the set defined by

$$
\sum_{j=1}^{n} p_{j}=0, \quad \sum_{j=1}^{k} p_{j} \in \bar{V}_{\mu}^{+}, \quad k=1, \ldots, n-1 .
$$

The asymptotic behavior of the truncated vacuum expectation values at spacelike infinity plays the major role in constructing the scattering states. Because of this, it is desirable 
to elucidate how the deformation under consideration affects this behavior. In accordance with (11), we define the deformed truncated $n$-point vacuum expectation values by

$$
w_{\iota_{1} \ldots \iota_{n}}^{T, \boldsymbol{\theta}}\left(f_{1} \otimes \cdots \otimes f_{n}\right) \stackrel{\text { def }}{=} w_{\iota_{1} \ldots \iota_{n}}^{T}\left(f_{1} \otimes_{\theta} \cdots \otimes_{\theta} f_{n}\right) .
$$

The usual method [29, 32] of estimating the spacelike asymptotic behavior of $w^{T}$, as well as the proof of cluster property (48) in [16], is based on Ruelle's auxiliary theorem which can be given the following form:

If two tempered distributions $u_{1}$ and $u_{2}$ coincide on an open cone $\Gamma$ and the supports of their Fourier transforms are separated by a finite distance, then both of these distributions vanishes at infinity faster than any inverse power of $|x|$ in any closed cone $G$ such that $G \backslash\{0\} \subset \Gamma$.

Indeed, for each test function $f \in S$, the convolution $\left(u_{1}-u_{2}\right) * f$ together with all its derivatives decreases rapidly in any direction within $\Gamma$, because a shift inside this cone implies that the test function moves away from support of $\left(u_{1}-u_{2}\right)$. The corresponding estimate is similar to that made in the proof of Lemma 1 for the case of test functions in $W_{\rho}^{\sigma} \subset S$. Let now $\chi(p)$ be a multiplier of $S$, equal to 1 on a neighborhood of supp $\hat{u}_{1}$ and equal to zero on a neighborhood of supp $\hat{u}_{2}$. Then we have the identity

$$
u_{1} * f=\left(u_{1}-u_{2}\right) *(\chi * f),
$$

which shows that $u_{1} * f$ also rapidly decreases inside $\Gamma$.

This theorem is applied to the truncated vacuum expectation values in the following way. Let $J$ be a nonempty subset of the set of indices $(1,2, \ldots, n)$ with a nonempty complement $J^{\prime}$. We let $\pi$ denote the permutation $(1,2, \ldots, n) \rightarrow\left(J, J^{\prime}\right)$ and $\pi^{\prime}$ denote the permutation $(1,2, \ldots, n) \rightarrow\left(J^{\prime}, J\right)$. By local commutativity, $w^{T}$ coincides with the permuted distribution $w_{\pi}^{T}$ on the cone

$$
\Gamma_{J}=\bigcap_{j \in J, j^{\prime} \in J^{\prime}} \Gamma_{j j^{\prime}}, \quad \text { where } \quad \Gamma_{j j^{\prime}}=\left\{x \in \mathbb{R}^{4 n}:\left(x_{j}-x_{j^{\prime}}\right)^{2}<0\right\},
$$

and $w_{\pi}^{T}$ in turn coincides with $w_{\pi^{\prime}}^{T}$ on this cone. It follows from the spectrum condition that $\hat{w}_{\pi^{\prime}}^{T}=0$ if $\sum_{j \in J} p_{j}=P_{J} \notin V_{\mu}^{+}$, and that $\hat{w}_{\pi}^{T}=0$ if $P_{J} \notin V_{\mu}^{-}$, because $P_{J}+P_{J^{\prime}}=0$ by the translation invariance. The cones $\Gamma_{J}$ with various $J$ cover the plane $x_{1}^{0}=\cdots=x_{n}^{0}$ in $\mathbb{R}^{4 n}$, and the Ruelle theorem says that, for any $f \in S\left(\mathbb{R}^{4 n}\right)$, the function $w_{\iota_{1} \ldots \iota_{n}}^{T} * f$ restricted to this plane and considered as a function of the difference variables $\mathbf{x}_{j}-\mathbf{x}_{j+1}$ belongs to $S\left(\mathbb{R}^{3(n-1)}\right)$. It is precisely the property of $w_{\iota_{1} \ldots \iota_{n}}^{T} * f$ that is used in [29, 32] to prove the existence of asymptotic scattering states. 
The Ruelle theorem can be considerably strengthened using the freedom in choosing the multiplier $\chi$ in (51). By varying this multiplier, the distributions $u_{1}$ and $u_{2}$ can be shown to decay exponentially inside $G$ with a rate constant determined by the distance between supp $\hat{u}_{1}$ and supp $\hat{u}_{2}$. In [33], this improvement is reduced to an extremum problem whose solution is expressed through Chebyshev polynomials. To detect this decay, appropriate test functions are needed. Clearly, they should decrease sufficiently fast at infinity, and we use the functions in $S$ that have exponential decrease of order $\geq 1$ and type $\geq 1 / l$, and satisfy the condition

$$
\|f\|_{N, \bar{l}} \stackrel{\text { def }}{=} \max _{|\kappa| \leq N} \sup _{x}\left|\partial^{\kappa} f(x)\right| \prod_{j} e^{\left|x_{j}\right| / \bar{l}}<\infty
$$

for all $\bar{l}>l$ and $N=0,1, \ldots$ Letting $S_{1, l}$ denote this space, the result [33] can be stated as follows. In local quantum field theory with the spectrum condition (49), the convolution of $w_{\iota_{1} \ldots \iota_{n}}^{T}$ with any test function $f \in S_{1, l}$ satisfies the estimate

$$
\left|\partial^{\kappa}\left(w_{\iota_{1} \ldots \iota_{n}}^{T} * f\right)(x)\right|_{x_{1}^{0}=\cdots=x_{n}^{0}} \leq C_{\bar{l}}\|f\|_{|\kappa|+K, \bar{l}} \exp \left\{-\frac{\mu R}{2(n-1)(1+3 \mu \bar{l})}\right\},
$$

where $\bar{l}>l$ and can be taken arbitrarily close to $l$, the constant $K$ is determined by the order of singularity of $w_{\iota_{1} \ldots \iota_{n}}^{T}$, and

$$
R=\max _{j, k}\left\|\mathbf{x}_{j}-\mathbf{x}_{k}\right\| .
$$

With $l \ll 1 / \mu$, (52) shows that $w_{\iota_{1} \ldots \iota_{n}}^{T}$ decays no slower than $\exp \{-\mu R / 2(n-1)\}$ as $R \rightarrow \infty$. In order to characterize the behavior of the deformed functions $w_{\iota_{1} \ldots \iota_{n}}^{T, \theta}$ at infinity, it is again necessary to choose the test functions in an adequate way. We use the spaces $W_{1,1 / l}^{\sigma, b} \subset S_{1, l}$, where $\sigma$ and $b$ can be taken arbitrarily large. This choice cannot be illustrated by the example of a free scalar field because its truncated $n$-point functions vanish identically, except for $n=2$, and the two-point function, being translation invariant, is unchanged by the deformation. But as a hint, we note that to test, e.g., the behavior of $\Delta_{+} \otimes_{\theta} \Delta_{+}$in the spacelike directions, it is natural to use test functions decreasing like $\exp \{-|x| / l\}$, with $l \ll 1 / \mu$, and whose Fourier transforms behave at infinity no worse, because the twisting of the tensor product intermixes the coordinate-space asymptotic behavior with that in momentum space, as shows (18) and the explanatory example given in Sec. IV]

Theorem 3 . If the assumption (49) on the existence of a mass gap holds, then for each test function $f \in W_{1,1 / l}^{\sigma, b}$, the function $w_{\iota_{1} \ldots \iota_{n}}^{T, \theta} * f$ satisfies the inequalities

$$
\left|\partial^{\kappa}\left(w_{\iota_{1} \ldots \iota_{n}}^{T, \theta} * f\right)(x)\right|_{x_{1}^{0}=\cdots=x_{n}^{0}} \leq C_{\kappa, \bar{l}}\|f\|_{1 / \bar{l}, \bar{b}} \exp \left\{-\frac{\mu R}{2(n-1)(1+3 \mu \bar{l})}\right\},
$$


where $\bar{l}>l$ and can be chosen arbitrarily close to $l$.

Proof. By definition (50), we have

$$
w_{\iota_{1} \ldots \iota_{n}}^{T, \theta} * f=w_{\iota_{1} \ldots \iota_{n}}^{T} * h, \quad \text { where } \quad \hat{h}(p)=\hat{f}(p) \prod_{j<k} e^{-\frac{i}{2} p_{j} \theta p_{k}} .
$$

The Fourier transformation maps $W_{1,1 / l}^{\sigma, b}$ onto the space of functions analytic in the complex $(1 / l)$-neighborhood of the real space and satisfying, for each $\bar{l}>l$, the conditions

$$
\sup _{|s| \leq 1 / \bar{l}}|g(p+i s)| \leq C_{\bar{l}, \bar{b}^{\prime}} \prod_{j} e^{-\bar{b}^{\prime}\left|p_{j}\right|^{\sigma^{\prime}}}, \quad \text { where }|s|=\max _{j}\left|s_{j}\right|
$$

Because $\sigma^{\prime}>1$, Lemma 2 obviously extends to this space, and the function $\prod_{j<k} e^{-\frac{i}{2} p_{j} \theta p_{k}}$ is hence its multiplier. Therefore, $h$ belongs to $W_{1,1 / l}^{\sigma, b}$ and depends continuously on $f$. It follows from the Cauchy theorem that the norms of $S_{1, l}$ and $W_{1,1 / l}^{\sigma, b}$ are related by $\|h\|_{N, \bar{l}} \leq$ $C_{N, \bar{l}}\|h\|_{1 / \bar{l}, \bar{b}}$. We conclude that if $f \in W_{1,1 / l}^{\sigma, b}$, then (52) implies (53), and Theorem 3 is thus proved.

Remark 5 . For simplicity, we have considered the vacuum expectation values of products of deformed fields $\prod_{j} \phi_{\iota_{j}}^{\theta}\left(x_{j}\right)$ with a common deformation parameter $\theta$. However, an analogous theorem holds for products $\prod_{j} \phi_{\iota_{j}}^{\theta_{j}}\left(x_{j}\right)$ with different $\theta_{j}$. The proof is the same, but with a multiplier of a different form than $\prod_{j<k} e^{-\frac{i}{2} p_{j} \theta p_{k}}$ in (154). As it is clear from the foregoing, the most interesting case is that when $\theta_{j}$ differ only in sign.

\section{CONCLUSION}

The noncommutative deformation (10) gives an interesting example of quantum fields defined as operator-valued tempered distributions on the Schwartz space $S$ and satisfying the asymptotic commutativity condition previously proposed for highly singular nonlocal fields with analytic test functions in $S^{\beta}=W^{1 /(1-\beta)}$, where $\beta<1$. It should be emphasized that the asymptotic commutativity principle [24, 26] is not fully implemented in the simplest deformation of Wightman field theory considered here, because the commutator $\left[\phi_{\iota}^{\theta}\left(x_{1}\right), \phi_{\iota^{\prime}}^{\theta}\left(x_{2}\right)\right]$ of fields with equal deformation parameters does not satisfy it. This commutator decreases in the spacelike region in the same fashion as the Wightman functions, i.e., exponentially with the damping factor depending on the threshold mass $\mu$. A more sophisticated way of deformation is apparently required for the deformed field theory to 
meet fully the condition of asymptotic commutativity and thus allow a consistent physical interpretation as nonlocal field theory.

Theorem 2 proved above can be supplemented by an additional statement. The noncommutative deformation (10) enters an elementary length $\ell \sim \sqrt{|\theta|}$ into the theory, and this length can be included in the characterization of the behavior of the matrix elements $u_{\Psi, \Phi}$ of the field commutator $\left[\phi_{\iota}^{\theta}\left(x_{1}\right), \phi_{\iota^{\prime}}^{-\theta}\left(x_{2}\right)\right]$. Namely, it can be shown that if the function $g$ in the definition (13) of the vector $\Phi$ belongs to $S^{\beta}\left(\mathbb{R}^{4 n}\right)$, where $\beta<1 / 2$, then the distribution $u_{\Psi, \Phi}$ has a continuous extension to the space $W^{2, b}(\mathbb{V})$, where $b=1 /\left(2 \ell^{2}\right)$. As proved in [22], such a property is also characteristic of the matrix elements of the commutator $\left[\phi\left(x_{1}\right), \phi\left(x_{2}\right)\right]$, where $\phi(x)$ is the deformed normal ordered square $: \varphi \star_{\theta} \varphi:(x)$ of a free scalar field $\varphi$.

Theorem 3 shows, in particular, that incoming and outgoing $n$-particle scattering states can be defined for the deformed interacting fields in four-dimensional spacetime in the usual way [29, 32] without appealing to the wedge locality. In the case of lower dimensions it should be combined with Hepp's idea [34] of using the so-called non-overlapping scattering states. In fact, to prove the existence of the $\theta$-dependent asymptotic states, it suffices to use a weaker version of Theorem 3 which employs test functions with compact support in momentum space and shows a decrease faster than any power of $1 / R$, but the strong version (53) is essential to understanding the analytic properties of the corresponding Smatrix. The construction of the scattering matrix is a more subtle and complicated problem which will be discussed in detail in a subsequent paper. A preliminary analysis shows that the arguments used for this purpose in [14, 15, 18] can be adapted to asymptotic commutativity. Then it makes sense to consider a wider class of deformations, not necessarily preserving the wedge locality. In this connection, it is worth noting that an analogous deformation can be performed on nonlocal quantum fields defined initially on $S^{\beta}$, where $\beta<1$, and subject to the asymptotic commutativity condition. Analogues of Theorems 2 and 3 can be shown to hold in this case too, and this implies the existence of asymptotic scattering states. 


\section{Acknowledgments}

This paper was supported in part by the the Russian Foundation for Basic Research (Grant No. 12-01-00865).

[1] S. Doplicher, K. Fredenhagen, and J. E. Roberts, "The Quantum structure of spacetime at the Planck scale and quantum fields", Commun. Math. Phys. 172 (1995) 187-220 [arXiv:hep-th/0303037].

[2] N. Seiberg and E. Witten, "String theory and noncommutative geometry", JHEP 09 (1999) 032 arXiv:hep-th/9908142.

[3] M. Chaichian, P. P. Kulish, K. Nishijima, and A. Tureanu, "On a Lorentz-invariant interpretation of noncommutative spacetime and its implications on noncommutative QFT", Phys. Lett. B 604 (2004) 98-102 arXiv:hep-th/0408069].

[4] J. Wess, "Deformed coordinate spaces; Derivatives", In: Mathematical, Theoretical and Phenomenological Challenges Beyond Standard Model (Proceedings of the BW2003 Workshop, 29 August-02 September 2003, Vrnjacka Banja, Serbia) (World Scientific, 2005) pp. 122-128 arXiv:hep-th/0408080.

[5] P. Aschieri, F. Lizzi, and P. Vitale, "Twisting all the way: From classical mechanics to quantum fields", Phys. Rev. D 77 (2008) 025037 arXiv:0708.3002].

[6] J. Zahn, "Remarks on twisted noncommutative quantum field theory", Phys. Rev. D 73 (2006) 105005 hep-th/0603231.

[7] A. P. Balachandran, T. R. Govindarajan, G. Mangano, A. Pinzul, B. A. Qureshi, and S. Vaidya, "Statistics and UV/IR mixing with twisted Poincaré invariance", Phys. Rev. D 75 (2007) 045009 hep-th/0608179.

[8] G. Fiore and J. Wess, "On "full" twisted Poincaré symmetry and QFT on Moyal-Weyl spaces", Phys. Rev. D 75 (2007) 105022 arXiv:hep-th/0701078.

[9] M. Riccardi and R. J. Szabo, "Duality and braiding in twisted quantum field theory," JHEP 01 (2008) 016 [arXiv:0711.1525].

[10] E. Joung and J. Mourad, "QFT with twisted Poincaré invariance and the Moyal product," JHEP 05 (2007) 098 arXiv:hep-th/0703245.

[11] A. P. Balachandran, A. Pinzul, and B. A. Qureshi, "Twisted Poincaré invariant quantum field theories," Phys. Rev. D 77 (2008) 025021 arXiv:0708.1779].

[12] G. Fiore, "Can QFT on Moyal-Weyl spaces look as on commutative ones?", Prog. Theor. Phys. Supplement, 171 (2007) 54-60 [arXiv:0705.1120].

[13] M. Chaichian, K. Nishijima, T. Salminen, A. Tureanu, "Noncommutative quantum field theory: A confrontation of symmetries", JHEP 06 (2008) 078 [arXiv:0805.3500].

[14] H. Grosse and G. Lechner, "Wedge-local quantum fields and noncommutative Minkowski space", JHEP 11 (2007) 012 arXiv:0706.3992].

[15] H. Grosse and G. Lechner, "Noncommutative deformations of Wightman quantum field theories," JHEP 09 (2008) 131 [arXiv:0808.3459]. 
[16] R. F. Streater and A. S. Wightman, PCT, Spin and Statistics and All That (Princeton Univ Press, Princeton, NJ, 2000).

[17] D. Buchholz, G. Lechner, and S. J. Summers, "Warped convolutions, Rieffel deformations and the construction of quantum field theories", Commun. Math. Phys. 304 (2011) 95-123 arXiv:1005.2656.

[18] G. Lechner, "Deformations of quantum field theories and integrable models", Commun. Math. Phys. 312 (2012) 265-302 arXiv:1104.1948.

[19] C. Dappiaggi, G. Lechner, and E. Morfa-Morales, "Deformations of quantum field theories on spacetimes with Killing fields", Commun. Math. Phys. 305 (2011) 99-130 arXiv:1006.3548].

[20] S. Alazzawi, "Deformations of fermionic quantum field theories and integrable models", Lett. Math. Phys. 103 (2013) 37-58 arXiv:1203.2058.

[21] M. Plaschke, "Wedge local deformations of charged fields leading to anyonic commutation relations", Lett. Math. Phys. 103 (2013) 507-532 [arXiv:1208.6141].

[22] M. A. Soloviev, "Failure of microcausality in noncommutative field theories", Phys. Rev. D 77 (2008) 125013 arXiv:0802.0997.

[23] M. A. Soloviev, "Noncommutative deformations of quantum field theories, locality, and causality", Theor. Math. Phys. 163 (2010) 741-752 arXiv:1012.3536

[24] M. A. Soloviev, "PCT, spin and statistics, and analytic wave front set", Theor. Math. Phys. 121 (1999) 1377-1396 arXiv:hep-th/0605243.

[25] M. A. Soloviev, "Star product algebras of test functions", Theor. Math. Phys. 153 (2007) 1351-1363 arXiv:0708.0811.

[26] V. Ya. Fainberg and M. A. Soloviev, "Nonlocalizability and asymptotical commutativity", Theor. Math. Phys. 93 (1992) 1438-1449 arXiv:hep-th/9211099].

[27] Y. Liao and K. Sibold, "Spectral representation and dispersion relations in field theory on noncommutative space", Phys. Lett. B 549 (2002) 352-361 [arXiv:hep-th/0209221].

[28] L. Álvarez-Gaumé and M. A. Vazquez-Mozo, "General properties of noncommutative field theories", Nucl. Phys. B 668 (2003) 293-321 arXiv:hep-th/0305093].

[29] N. N. Bogolyubov, A. A. Logunov, A. I. Oksak, and I. T. Todorov, General Principles of Quantum Field (Kluwer, Dordrecht, 1990).

[30] I. M. Gelfand and G. E. Shilov, Generalized Functions, (Academic, New York, 1967), Vol. 3.

[31] M. A. Soloviev, "Nonlocal extension of the Borchers classes of quantum fields", In: Multiple Facets of Quantization and Supersymmetry. Michael Marinov Memorial Volume, tdited by M. Olshanetsky and A. Vainshtein (World Scientific, Singapore, 2002), pp. 697-717 arXiv:math-ph/0112053.

[32] R. Jost, The General Theory of Quantum Fields (American Math. Soc., Providence, RI, 1965).

[33] M. A. Solovev, "A generalization of Ruelle's theorem", Theor. Math. Phys. 52 (1982) 756-763.

[34] K. Hepp, "On the connection between Wightman and LSZ quantum field theory", Commun. Math. Phys. 1 (1965) 95-111. 